This document is confidential and is proprietary to the American Chemical Society and its authors. Do not copy or disclose without written permission. If you have received this item in error, notify the sender and delete all copies.

\title{
Structural and mechanistic basis for anaerobic ergothioneine biosynthesis
}

\begin{tabular}{|r|l|}
\hline Journal: & Journal of the American Chemical Society \\
\hline Manuscript ID & ja-2018-12596j.R2 \\
\hline Manuscript Type: & Article \\
\hline Author: & $29-$ Mar-2019 \\
\hline Complete List of Authors: & $\begin{array}{l}\text { Leisinger, Florian; University of Basel, Department for Chemistry } \\
\text { Burn, Reto; University of Basel, Department for Chemistry } \\
\text { Meury, Marcel; Five Prime Therapeutics } \\
\text { Lukat, Peer; Helmholtz Centre for Infection Research, Molecular } \\
\text { Structural Biology } \\
\text { Seebeck, Florian; University of Basel, Department for Chemistry }\end{array}$ \\
\hline
\end{tabular}

\section{SCHOLARONE ${ }^{m}$ Manuscripts}


Sulfur plays a central role in the primary and secondary metabolism of any organism. The availability of numerous redox states and the high polarizability enable sulfur to react as nucleophile, as electrophile or as radical, and make this element an irreplaceable building block in many small and large

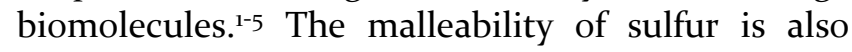
reflected in the broad range of different enzymatic reactions that form carbon-sulfur (C-S) bonds. ${ }^{1}$ Because of this diversity it is not uncommon that natural products with similar sulphurous substructures emerge from entirely different chemistries. For example, the thiazolidine moiety of penicillin is formed in an oxygen-dependent reaction catalysed by an iron-dependent oxidase, ${ }^{6}$ whereas the

thiophane ring in biotin is formed by an S-adenosyl methioneine dependent radical enzyme. ${ }^{7}$

Even more remarkable is the case of thiamine biosynthesis where bacteria, archaea and eukaryotes utilize different $\mathrm{C}-\mathrm{S}$ bond forming reactions to produce an identical product. ${ }^{8-10}$

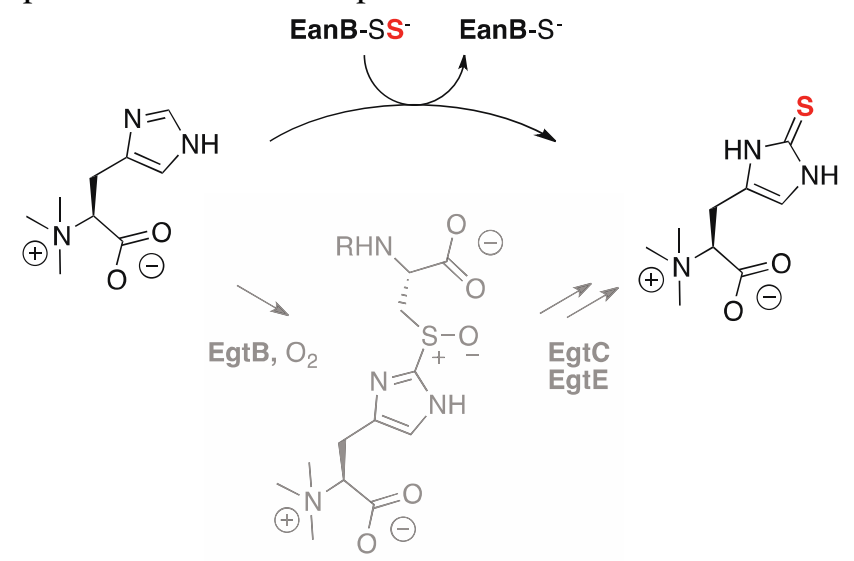

Figure 1. The rhodanese-like enzyme EanB from Chlorobium limicola catalyzes oxidative sulfur transfer to Na-trimethylhistidine (TMH) in an oxygenindependent reaction." The sulfoxide synthase EgtB from Mycobacterium thermoresistibile catalyzes oxygen-dependent sulfurization of TMH. ${ }^{12}$ This second pathway requires two additional enzymes (EgtC and E) to produce ergothioneine. ${ }^{13-14}$

Recently, we discovered that ergothioneine biosynthesis also emerged through multiple routes."1,

15 Actinobacteria, cyanobacteria, many proteobacteria and most fungi synthesize ergothioneine by way of an iron-dependent sulfoxide synthase (EgtB, Figure 1). In cooperation with two additional enzymes EgtB attaches a cysteine-derived sulfur atom to the imidazole ring of $\mathrm{N} \alpha$-trimethyl histidine (TMH). ${ }^{16-18}$ Although the mechanistic 
details of the EgtB reaction are controversial, the involvement of oxygen as an electron acceptor is undisputed. ${ }^{12}$, 19-20 In contrast, a group of mainly anaerobic bacteria and archaea recruited a rhodanese-like enzyme (EanB) to introduce sulfur into TMH. ${ }^{11}$ Rhodanese-like enzymes are ubiquitous C-S bond forming catalysts involved in the biosynthesis of cofactors, thiosugars and thiolated nucleic acids., 8, 21-23 Invariably, these enzymes bind their substrate in activated

\section{A}
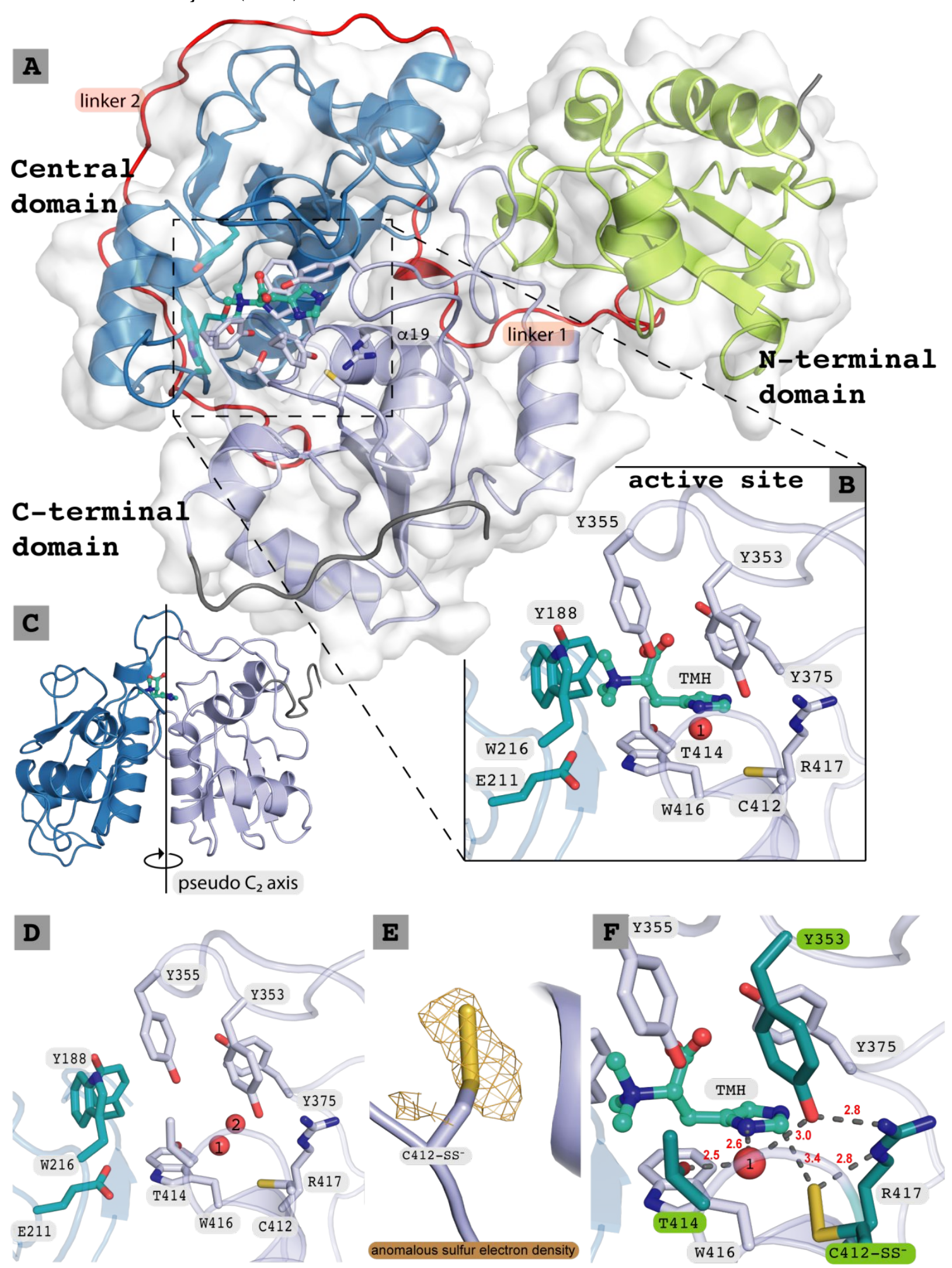
Figure 2. A: Overall view of EanB in complex with TMH (turquoise). Active site residues are shown in lilac and turquoise-blue, $\mathrm{N}$-terminal domain in green, central domain in blue, $\mathrm{C}$-terminal domain in violet, linker regions in red. B: active site of TMH complexed EanB structure with residues represented as sticks, $\mathrm{H}_{2} \mathrm{O}_{-} 1$ is shown a red sphere. C: pseudo $\mathrm{C}_{2}$ symmetry axis between central and C-terminal domain. D: active site of apo-EanB. $\mathrm{H}_{2} \mathrm{O}_{-} 1$ hydrogen bonds to a second water molecule instead of TMH. E: anomalous sulfur electron density map at catalytic cysteine persulfide (Cys412-SS) revealed presence of two sulfur atoms. F: Active site model of the reactive complex with TMH and Cys-SS. Distances indicated in red.

form, after phosphorylation or adenylylation, and mediate nucleophilic substitution of the phosphate or the AMP leaving group with a sulfur atom. EanB does not follow this paradigm. This enzyme binds its substrate $\mathrm{TMH}$ without prior activation and attaches a sulfur atom in place of a proton. This combination of substrate activation with oxidative sulfurization is unprecedented in enzymology. ${ }^{1}$

To elucidate the catalytic mechanism of EanB we solved the crystal structure of this enzyme and we examined the catalytic activity of this enzyme and variants thereof. The described structural and kinetic observations suggest that C-S bond formation occurs by nucleophilic attack of an active site persulfide anion onto the imidazolium ring of TMH. Significant active site similarity among a large subclass of rhodanese-like enzymes with unknown functions indicates that this reaction type may apply to several different biosynthetic pathways occurring in proteobacteria, spirochaetes and firmicutes.

\section{Results}

Crystal structure of native EanB. We produced EanB from Chlorobium limicola in Escherichia coli as previously described." To optimize EanB for crystallization we engineered a truncated version of this protein that lacks the first 34 residues. A homology model of EanB based on the crystal structure of the rhodanese-like enzyme YnjE suggested that this $\mathrm{N}$-terminal appendix is not part of the folded core and is located far away from the presumed active site. ${ }^{24}$ In addition, we mutated three consecutive lysine residues on the protein surface to alanine ( $\mathrm{K}_{357} \mathrm{~A}, \mathrm{~K}_{35} 8 \mathrm{~A}$ and $\left.\mathrm{K}_{359} \mathrm{~A}\right)$ in order to reduce the surface entropy (Figure $\mathrm{S1}$ ). ${ }^{25}$ The resulting protein $\left(\operatorname{EanB}_{\mathrm{SER}}\right)$ is stable, active (Table 1) and formed diffracting crystals (Figure S2). One diffraction data set with a resolution of $1.8 \AA$ was used to solve the structure of EanB by molecular replacement (Table S1). ${ }^{26}$ The measured electron density revealed a continuous polypeptide chain from Glu35 to Pro456. The residue numbering was chosen in accord with the gene locus Clim_1149 in the genome of Chlorobium limicola DSM 245. EanB folds into three consecutive rhodanese-like domains (35-139, 160-291, 319 - 445) which are connected by 19 and 26 residue long linker regions (red, Figure 2A). The structure of EanB is remarkably similar to the $E$. coli protein YnjE (Figure 3A).24 A detailed description of the overall structure of EanB can be found in the supporting information (Figure $\mathrm{S}_{3}$ and $\mathrm{S}_{4}$ ). The central- and Cterminal domain form a dimer with pseudo $\mathrm{C}_{2}$ symmetry (Figure $2 \mathrm{C}$ ). The active site - as identified by the location of a conserved cysteine residue in the third domain, ${ }^{24}$ maps to the dimer interface near the pseudo $\mathrm{C}_{2}$-symmetry axis. The $\mathrm{N}$-terminal docks to this dimer far away from the active site and possibly serves as a stabilizing clamp. One conspicuous difference between EanB and YnjE occurs near the active site. EanB residues 245 - 260 and $348-360$ form two loops that shape the active site into a deep and narrow tunnel with the catalytic cysteine at its bottom. YnjE lacks the first loop leaving the active site as a large and water exposed cleft (Figure 3). ${ }^{24}$

Structure of EanB in complex with TMH. Based on a data set with a resolution of $2.8 \AA$ (Table $\mathrm{S}_{1}$ ) we also obtained the structure of EanB in complex with the substrate TMH. This structure provides the first view at a triple-domain rhodanese-like enzyme bound to its genuine substrate (Figure $2 \mathrm{~B}$, Figure $\mathrm{S}_{5}$ ). TMH stacks on top of the indole ring of Trp416. $\mathrm{N} \tau$ and $\mathrm{N} \pi$ of the imidazole ring hydrogen bond to the backbone carbonyl of Ala374 (2.8 A, Figure S9) and to a water molecule $\left(\mathrm{H}_{2} \mathrm{O} \_1,2.6 \AA\right)$ respectively. This water molecule is immobilized by two additional hydrogen bonds to the side chains of Thr414 (2.5 ̊) and Tyr353 (3.0 ̊). The backbone amides of residues $414-416$ point three additional hydrogen bond donors towards $\mathrm{H}_{2} \mathrm{O} \_1$ (3.0-3.5 $\AA$ ). The carboxylate group of TMH is solvated by the phenol functions of Tyr375 and Tyr355 (Figure S6). The N $\alpha$-trimethyl ammonium moiety docks into an aromatic box formed by the side chains of Tyri 88 , Trp216, Trp416, Tyr355. The side chain of Glu178 points towards this aromatic pocket and presumably neutralizes the positive charge of the ammonium ligand. In the apo structure this ammonium binding pocket hosts a loosely 
coordinated magnesium cation, corroborating the cation-binding propensity of this site. It is unlikely that this cation plays any role in EanB catalysis. Similar aromatic boxes are typical for betaine binding proteins. ${ }^{27-29}$

Structure of the persulfide form of EanB. A third data set with a resolution of $1.6 \AA$ revealed the structure of EanB with extra electron density around the active site cysteine Cys412 (Figure 2E). This protrusion fits well to a model with a sulfane sulfur attached to Cys412. A point atom refined model revealed a distance of the electron density maximum centers between Cys-S $\gamma$ and the protrusion (point atom) of $2.04 \AA$. This distance is in good agreement with the expected length of an S-S bond $(2.07 \AA) .3^{30}$ In contrast, the S-O bond of a cysteine sulfenic acid is much shorter ( $1.67 \AA$ ), and produced no acceptable fit upon model refinement (Figure $\mathrm{S}_{7}$ ). Further evidence for the presence of a persulfide function at Cys412 was gained from anomalous diffraction data sets. Restrained SAD data refinement revealed a peak in anomalous sulfur electron density map corresponding to two sulfur atoms at this position (Figure $2 \mathrm{E}$ ). The presence of persulfide function is corroborated by the observation that recombinant EanB can convert up to one equivalent of

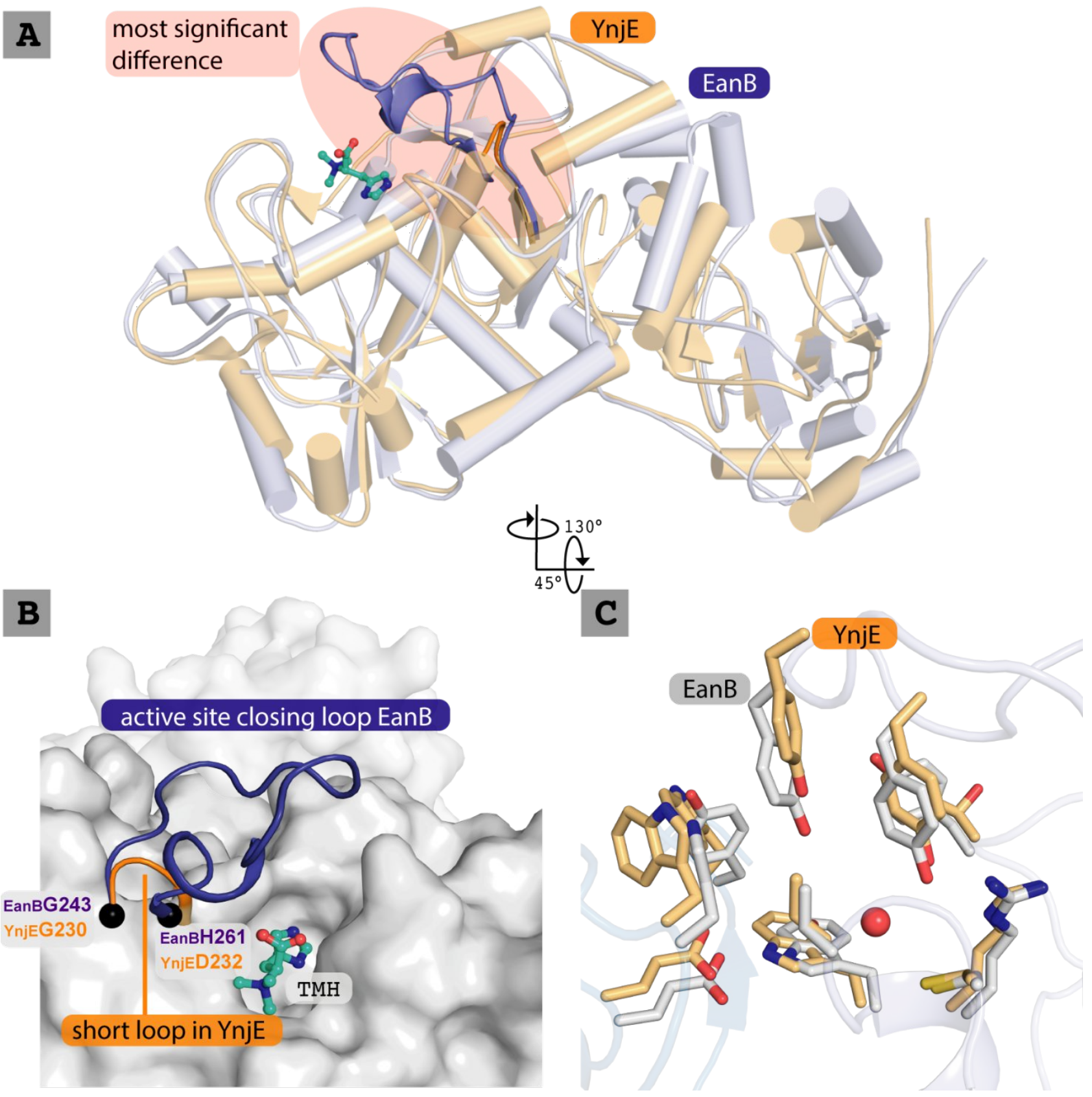


Figure 3. A: Superposition of EanB structure with YnjE in simplified ribbon representation (PDB code 3IPO, RMDS: $2.64 \AA$ over 400 residues). ${ }^{24}$ B: the extra active site loop in EanB is shown in dark blue (residues $245-260$ ). The much shorter turn in YnjE is shown in orange. C: superposition of active site residues of EanB with YnjE.

TMH to ergothioneine in the absence of exogenous sulfur donor. "The persulfide is in hydrogen bonding distance to the guanidinium side chain of Arg417 (2.8 A, Figure 2F). The sulfane sulfur atom approaches Arg417 in the plane of the guanidinium function (dihedral angle NCNS: $-8.6^{\circ}$, bond angle CNS: $120^{\circ}$ ). This geometry is suggestive of hydrogen bonding. The entire persulfide is placed in the positive electrostatic field generated by the dipole moment of one of the two symmetry-related $\alpha$ helices (residues $415-429$ ). In the absence of the sulfane sulfur atom, the side chain of Cys412 adopts an identical conformation placing the side chain in hydrogen bonding distance to Gly415 (3.2 $\AA$ ) and to H2O_1 (3.3 $\AA$ ).

Model of the reactive complex. Comparison of the native structure of EanB, the persulfide form and the complex with TMH shows that substrate binding induces almost no conformational change in the active site (Figure S8, Table S2). Therefore, we concluded that superposition of the persulfide form and the TMH complex should provide a relevant model for the reactive complex of EanB (Figure $2 \mathrm{~F}$ ). In this model the sulfane sulfur on Cys412 approaches TMH from below the imidazole plane to a near Van der Waals distance $\left(\mathrm{S}_{\mathrm{Cys} 412}-\right.$ $\mathrm{C}_{2 \mathrm{TMH}}, 3.4 \AA$ ). The phenol ring of Tyr353 hoovers on the other side of the imidazole plane in contact with Arg417 (2.8 ̊), $\mathrm{H}_{2} \mathrm{O} \_1$ (3.0 ̊̊), and the backbone of Gly413 (3.3 $\AA$ ).

Mutagenesis of active site residues. The surface of the active site is formed by ten residues. Although six out of the ten residues are aromatic, polar interactions are clearly most important for binding and catalysis. To examine these interactions we constructed four EanB variants that each lack one hydroxyl group by mutating Thr414 to Ala and Tyr353, Tyr 355 or Tyr375 to Phe (Table 1).

\begin{tabular}{l|cccc} 
Table 1 ${ }^{[\mathrm{a}]}$ \\
\hline Enzyme & $\begin{array}{c}k_{\text {turnover }} \\
{\left[\mathrm{s}^{-1}\right]}\end{array}$ & $\begin{array}{c}k_{\mathrm{cat}} \\
{\left[\mathrm{s}^{-1}\right]}\end{array}$ & $\begin{array}{c}K_{\mathrm{M}, \mathrm{TMH}} \\
{[\mathrm{M}]}\end{array}$ & $\begin{array}{c}k_{\text {turnover }} / \\
K_{\mathrm{M}} \\
{\left[\mathrm{M}^{-1} \mathrm{~s}^{-1}\right]}\end{array}$ \\
& $\mathrm{x} \mathrm{10}^{-3}$ & $\mathrm{x} \mathrm{10}^{-3}$ & $\mathrm{x} \mathrm{10}^{-6}$ & ${ }^{-6}$ \\
\hline EanB $_{\mathrm{wt}}$ & $8.3^{[\mathrm{b}]}$ & 11 & 40 & 275 \\
EanB $_{\text {SER }}$ & 8.5 & n.d. & n.d. & n.d.
\end{tabular}

\begin{tabular}{l|cccc}
$\operatorname{EanB}_{\mathrm{T}_{414} \mathrm{~A}}$ & $<0.1$ & n.d. & n.d. & n.d. \\
$\operatorname{EanB}_{\mathrm{Y}_{333} \mathrm{~F}}$ & $<0.1$ & n.d. & n.d. & n.d. \\
$\operatorname{EanB}_{\mathrm{Y}_{355} \mathrm{~F}}$ & 6.5 & 5.0 & 4900 & 1.0 \\
$\operatorname{EanB}_{\mathrm{Y}_{375} \mathrm{~F}}$ & 5.5 & 2.5 & 1700 & 1.5 \\
\hline
\end{tabular}

${ }^{[a]}$ All values represent averages of at least two independent measurements. The standard error on all kinetic parameters is $\leq 20 \%$ of the average value. ${ }^{[b]}$ Data from Ref. ${ }^{11} .{ }^{[b]}$ n.d. $=$ not determined.

We excluded Arg417 from this study, because mutagenesis of active site arginines to any other proteinogenic amino acid is usually too disruptive to give interpretable results.

The catalytic activities of these variants $\left(\operatorname{EanB}_{\mathrm{T}_{414} \mathrm{~A}}\right.$, EanB $_{\mathrm{Y}_{353} \mathrm{~F}}$, EanB $\left.\mathrm{B}_{3555} \mathrm{~F}, \operatorname{EanB}_{\mathrm{Y}_{375} \mathrm{~F}}\right)$ were assessed by measuring the rate of ergothioneine production in single turnover reactions. For this characterization we exploited the fact that EanB can be isolated partially in persulfide form from E. coli lysates. ${ }^{11}$ The persulfide content of the EanB variants was also determined by colorimetric quantification of thiocyanide formed when the proteins were incubation with cyanide (Table $\mathrm{S}_{3}$ ). This analysis showed that all isolated EanB variants contained between 0.2 to 0.5 equivalent sulfane sulfur.

To determine single-turnover rates $\left(k_{\text {turnover }}\right)$, we incubated the enzymes with a saturating concentration of TMH and monitored product formation by HPLC. In this assay $\operatorname{EanB}_{\mathrm{wt}}, \operatorname{EanB}_{\mathrm{SER}}$, EanB $_{\mathrm{Y}_{355}}$ and EanB $\mathrm{Y}_{375}$ catalyzed sulfur transfer to $\mathrm{TMH}$ at similar rates (Table 1, Figure Sio). In contrast, no product formation was observed for EanB $_{\mathrm{T}_{414} \mathrm{~A}}$ and EanB $_{\mathrm{Y}_{353} \mathrm{~F}}$, suggesting that their activity is less than $1 \%$ of the wild type activty. This dramatic effect establishes Thr414 and Tyr353 as essential catalytic residues. The available kinetic data does not allow a judgement as to whether the mutations predominantly affected substrate binding or turnover. However, since these residues only make indirect or weak interactions with $\mathrm{TMH}$, it is save to assume that the mutations predominantly affected turnover.

To interrogate the functions of Tyr355 and Tyr375 in more detail, we determined the Michaelis-Menten parameters for $E \mathrm{AnB}_{\mathrm{wt}}, \mathrm{EanB}_{\mathrm{Y}_{355} \mathrm{~F}}$ and $\mathrm{EanB}_{\mathrm{Y}_{375} \mathrm{~F}}$ by measuring $k_{\text {turnover }}$ as a function of the TMH concentration (Figure $\mathrm{S}_{11}$ and $\mathrm{S} 12$ ). This experiment 
showed that $\operatorname{EanB}_{\mathrm{Y}_{355}}$ and $\mathrm{EanB}_{\mathrm{Y}_{375}}$ are characterized by a $K_{\mathrm{M}}$ values 170 and 60 -fold higher than that determined for wild type. Meanwhile, the catalytic constant $\left(k_{\text {cat }}\right)$ remained largely unaffected by either mutation. These observations suggest that Tyr355 and Tyr375 are mainly important for substrate recognition.

pH dependence of catalysis. The active site of EanB and the substrate TMH contain several ionizable groups with $\mathrm{p} K_{\mathrm{a}} \mathrm{s}$ close to the reaction $\mathrm{pH}$ (8.o). Therefore, we found it important to determine the $\mathrm{pH}$-dependence of EanB (Figure S12). This experiment showed that increasing the reaction $\mathrm{pH}$ from 5 to 9 reduced the $K_{\mathrm{M}}$ by 20 -fold and $k_{\text {turnover }}$ only by 1.3 -fold. Plotting the catalytic efficiency against $\mathrm{pH}$ revealed that the change in activity is related to a single protonation event characterized with a kinetic $\mathrm{p} K_{\mathrm{a}}$ of $6.1 \pm$ o.1. Because $k_{\text {turnover }}$ is not affected, it is clear that this protonation affects an equilibrium reaction before the rate limiting step. Since the imidazole $\mathrm{p} K_{\mathrm{a}}$ of TMH as inferred by NMR titration is $6.0 \pm 0.1$ (Figure $\mathrm{S}_{13}$ ), we interpret the $\mathrm{pH}$ dependence as evidence that EanB binds TMH only in neutral (deprotonated) form. Hence, lowering the reaction $\mathrm{pH}$ reduces the effective substrate concentration and in turn increase the observed $K_{\mathrm{M}}$. The absence of additional kinetically relevant protonation equilibria suggest that catalytic residues such as Tyr353 and Cys412 do not change their protonation state between $\mathrm{pH} 5$ and 9 .

Kinetic isotope effects. To probe which elementary step in the catalytic cycle of EanB may be rate limiting we measured substrate and solvent kinetic isotope effects (KIE). We have shown earlier that the rate limiting step must occur during sulfur transfer from EanB to TMH, rather than during sulfur transfer to EanB." To examine whether abstraction of the proton from the TMH imidazole ring at $\mathrm{C}_{2} \quad\left(\mathrm{C}_{2}-\mathrm{H}\right)$ may be rate limiting, we determined the substrate KIE on $k_{\text {turnover }}$ using $\mathrm{C}_{2}$ deuterated TMH as a substrate (Figure $\mathrm{S}_{4}$ ). This experiment revealed a KIE of $1.1 \pm 0.3$, suggesting that deprotonation of $\mathrm{C}_{2}$ is not rate limiting. Proton exchange at the $\mathrm{C}_{2}$ center of imidazole rings at $\mathrm{pH}$ 8.0 is slow enough that we can exclude nonenzymatic wash-out of the deuterium label from TMH as an explanation for the observed KIE. ${ }^{31}$

In contrast, we observed a significant inverse solvent KIE effect on $k_{\text {turnover }}(\mathrm{sKIE}=0.8 \pm 0.04$, Figure $\mathrm{S}_{15}$ ). The observation that the sKIE is not larger than unity provides evidence against a mechanism in which an exchangeable proton is being transferred during the rate limiting step.

\section{Discussion}

EanB-catalyzed sulfur transfer to TMH is formally an oxidation reaction that couples C-S bond formation with S-S bond reduction. This reaction type is also interesting because both the imidazole ring of TMH and cysteine persulfide can in principle act as nucleophile or as electrophile, depending on their protonation state. In the following we will match structural and kinetic evidence with different mechanistic proposals. As a result, we find that EanB-catalyzed sulfurization of TMH most likely occurs via nucleophilic attack of a persulfide anion onto the imidazolium ring of $\mathrm{TMH}$. Subsequently, we will summarize the indications that this reaction type may be catalyzed by many YnjE-like rhodaneses. ${ }^{24}$

Catalytic mechanism. Rhodanese and most rhodanese-like enzymes can transfer sulfur to cyanide to form thiocyanide. ${ }^{32}$ This reaction is facilitated by the considerable nucleophilicity of cyanide anion and the acidity of hydrogen cyanide $\left(\mathrm{p} K_{\mathrm{a}}=9\right) .33$ By analogy we surmised that EanB could deprotonate $\mathrm{TMH}$ at $\mathrm{C}_{2}$ to form the corresponding carbene which may attack the Cys412 persulfide (1a, Scheme 1). This scheme would require deprotonation of the imidazole or imidazolium ring of TMH. Although $\mathrm{C}_{2}$ protons in imidazolium rings are not particularly acidic $\left(\mathrm{p} K_{\mathrm{a}}=24\right), 3^{11}$ the conjugated base, the imidazole-2-yl carbene, does occur as an intermediate during proton exchange in water. ${ }^{31}$ The involvement of carbene intermediates in enzyme catalysis is also precedented by the cofactor chemistry of thiamine pyrophosphate. $34-35$ However, formation of a TMH carbene in the EanB reaction is unlikely. Proton abstraction would require a strong base positioned in plane with the imidazole ring, juxtaposed to the scissile $\mathrm{C}_{2}-\mathrm{H}$. The two candidate bases, Tyr353 and the persulfide anion of Cys412 are both located out of plane (Figure $2 \mathrm{~F}$ ). In addition, both moieties are weak bases (Tyr: $\mathrm{p} K_{\mathrm{a}}=10$; persulfide: $\left.6-7\right)^{33}$ and both make direct hydrogen bonding contact with the cationic side chain of Arg417. This interaction likely reduces rather than increases the basicity of Tyr353 and Cys412. As discussed below, the $\mathrm{pH}-$ dependence of EanB indicates that Tyr353 is protonated at physiological $\mathrm{pH}$ and is therefore not available as a base. The persulfide function of 
Cys412 is likley anionic. However, in a hypothetical equilibrium where $\mathrm{TMH}$ and the active site persulfide anion share a proton $\left(\mathrm{C}_{2}-\mathrm{H}\right)$, only one out of $10^{17}$ enzymes would be in the in the carbene state (1a, $\left.K_{\mathrm{a}, \mathrm{TMH}} / K_{\mathrm{a} \text {,persulfide }}=10^{-24} \mathrm{M} / 10^{-7} \mathrm{M}=10^{-17}\right)$. Since the enzyme turns over at a rate of $10^{-2} \mathrm{~s}^{-1}$ (Table 1 ), the carbene species ra would have to react forward at a rate equal or faster that $10^{15} \mathrm{~s}^{-1}$. This frequency is three orders of magnitude higher than that of many bond vibrations. Therefore we conclude that 1a is not kinetically competent. We have no evidence that the acidities of TMH or the persulfide are perturbed to the extent that this conclusion would not hold.

A second possible mechanism could be nucleophilic attack at the sulfane sulfur of Cys412 by the neutral imidazole ring of TMH (2a). Precedence for nucleophilic attack by an imidazole ring can be found in the mechanism of urocanases. ${ }^{36}$ In this reaction the imidazole ring of urocanic acid attacks the nicotinamide ring of $\mathrm{NAD}^{+}$. In EanB, nucleophilic attack by TMH is unlikely for the following reasons: The persulfide function of Cys412 is not protonated at physiological $\mathrm{pH}$. The low intrinsic $\mathrm{p} K_{\mathrm{a}}$ of persulfides $\left(\mathrm{p} K_{\mathrm{a}}=6.2\right), 33$ the close interaction between Cys412 and $\mathrm{Arg} 417$, and the $\mathrm{pH}-$ dependence of EanB all point to an anionic persulfide function. We note that proton transfer from Tyr 353 (Figure $2 \mathrm{~F}$ ) could provide a mechanism to activate the persulfide as an electrophile.

An even more severe problem of mechanism 2 is that intermediate $\mathbf{2 a}$ would require coordination by two hydrogen bond donors. Although the backbone carbonyl of Ala374 is in hydrogen-bonding distance to $\mathrm{N} \tau(2.8 \AA)$ this residue can hardly serve as a proton donor (Scheme 1, Figure S9). The remaining surface around $\mathrm{N} \tau$ is composed of hydrophobic moieties of Ile262, Ala259, Trp416, Arg417 and Tyr375, suggesting that this pocket is poorly equipped to stabilize intermediate $\mathbf{2 a}$.

The third model suggests that the nucleophilic persulfide anion attacks TMH (3b) to form $3 \mathbf{c}$. Tyr353 mediated $\mathrm{C}_{2}-\mathrm{H}$ cleavage from this intermediate leads to aromatization of the mercaptoimidazole ring and S-S bond cleavage (3d). The crystal structure of EanB, showing the suggested nucleophile and base positioned on opposite sides of the imidazole plane (Figure 3), is consistent with this mechanism. The observed $\mathrm{pH}$ dependence and kinetic isotope effects of EanB allow us to add further details to this mechanistic picture. The $K_{\mathrm{M}}$ of EanB is characterized by a kinetic $\mathrm{p} K_{\mathrm{a}}$ that is identical to the thermodynamic $\mathrm{p} K_{\mathrm{a}}$ of TMH. This dependence shows that EanB binds its substrate only in neutral form. There is no evidence for additional kinetically relevant protonation equilibria, suggesting that the protonation states in the active site - specifically those of Tyr353 and Cys412 - remain unchanged between $\mathrm{pH} 5$ and 9 . Therefore we conclude that species $3 a$ is the dominant first intermediate. Direct formation of $\mathbf{3} \mathbf{b}$, by encounter of cationic TMH and EanB with an anionic Tyr353 maybe possible but rate.

The proposition that Tyr353 is the proton donor to $\mathrm{TMH}$ is supported by the following considerations. Because an imidazolium ring is significantly more electrophilic than an imidazole ring, protonation would activate TMH for C-S bond formation. This proton must be donated by $\mathrm{H}_{2} \mathrm{O}_{-} 1$ (Figure $2 \mathrm{~F}$ ) because this is the only 
Mechanism 1

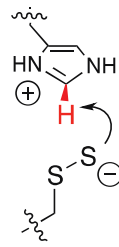

Mechanism 2

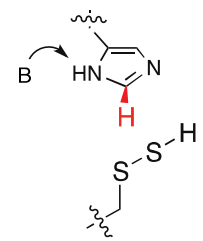

Mechanism 3
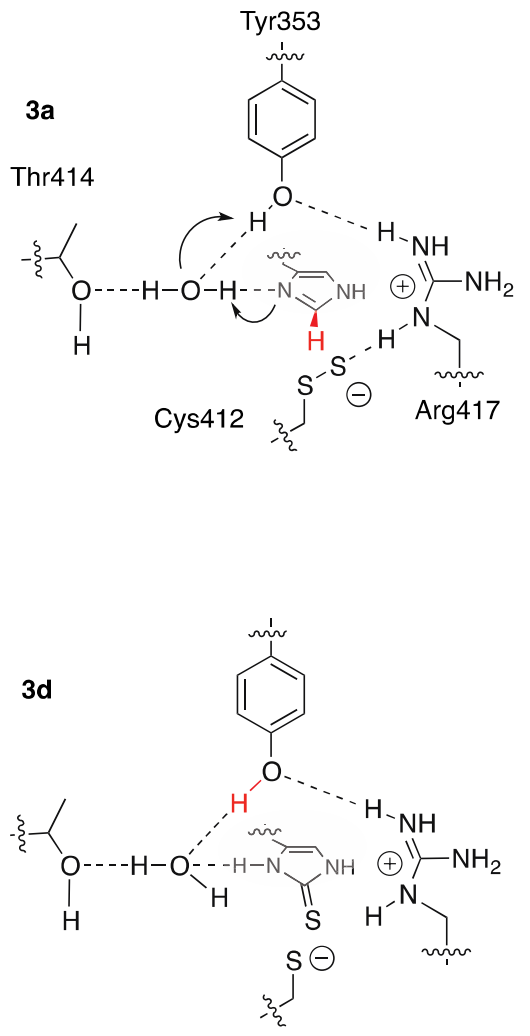

Scheme 1. Three possible catalytic mechanism of EanB.

possible hydrogen bond donor in direct contact with the imidazole ring of TMH. Since water has a much higher $\mathrm{p} K_{\mathrm{a}}$ than an imidazolium cation, formation of a hydroxide:imidazolium ion pair would produce a very unstable intermediate. Alternatively, $\mathrm{H}_{2} \mathrm{O} \_1$ could relay a proton from a secondary proton donor. Possible candidates are Thr414 and Tyr353. The latter is certainly more acidic. Proton transfer from Tyr353 to TMH would also activate Tyr353 as a base for subsequent $\mathrm{C}_{2}-\mathrm{H}$ cleavage. Hence, the most likely interpretation of the structure and $\mathrm{pH}$-dependence of EanB is that TMH and Tyr353 share a proton via $\mathrm{H}_{2} \mathrm{O}_{-} 1$ in the reactive complex.
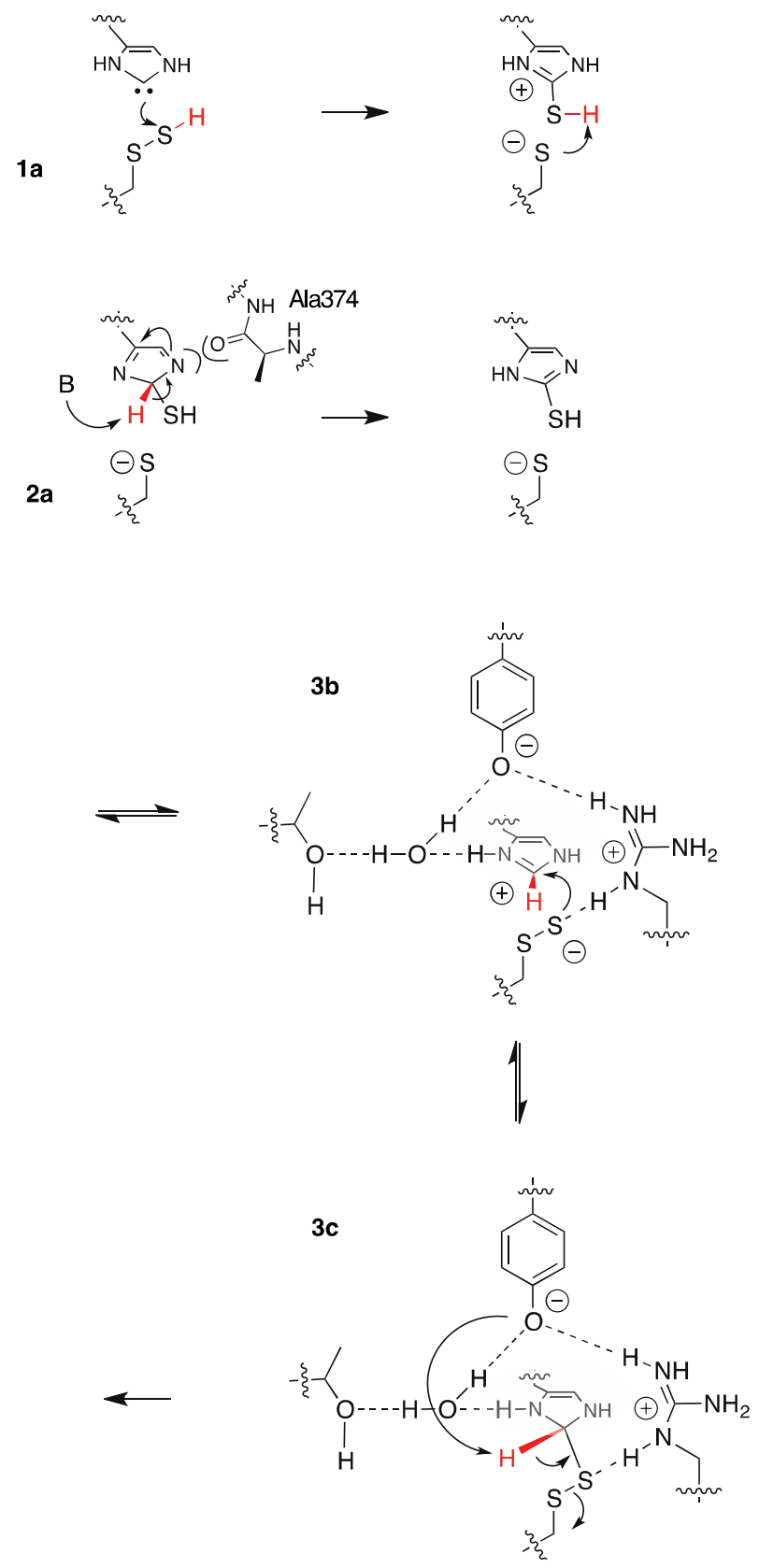

A second interesting question is whether protonation of TMH occurs in an equilibrium between $3 \mathbf{a}$ and $\mathbf{3} \mathbf{b}$, or whether $3 \mathbf{a}$ reacts directly to 3c via proton transfer coupled with C-S bond formation. The substrate KIE is close to unity, suggesting that proton abstraction from species $3 \mathbf{C}$ is not rate limiting. Since $k_{\text {turnover }}$ was measured in a single-turnover assay we can exclude product release as a rate limiting step. Since EanB turns over less than once every minute, substrate binding is also unlikely to be rate limiting. The remaining candidate for the rate limiting step is C-S bond formation. If this step is coupled to proton transfer from Tyr353 to TMH one might predict a solvent KIE significantly larger than one. In contrast, we 
found a slightly inverse KIE. This behavior does not provide evidence for coupled proton transfer and C$\mathrm{S}$ bond formation, but we cannot definitively exclude this mechanism either. An alternative interpretation is that proton transfer between TMH and Tyr353 occurs as an equilibrium connecting species $3 \mathbf{a}$ with $\mathbf{3} \mathbf{b}$. Because the $\mathrm{p} K_{\mathrm{a}}$ of the TMH imidazole ring is lower than that of Tyr353 - even in the enzyme complex, species $3 a$ is likely more stable than $\mathbf{3 b}$. Since $\mathbf{3} \mathbf{b}$ is immediately connected to the rate limiting step, the position of this internal equilibrium would directly factor into the rate of catalysis. This interpretation can also accommodate the the observed inverse KIE. It is possible that solvent deutration affects the equilibrium between $3^{\mathbf{a}}$ and $\mathbf{3} \mathbf{b}$ causing faster turnover in $\mathrm{D}_{2} \mathrm{O}$ than in water.

The reaction according to mechanism 3 is deceptively similar to that of nucleophilic aromatic substitution. Examples of proteins that attack electron-deficient heterocycles with S-nucleophiles include glutathione-S-transferases, 37 dimethylarginine dimethylaminohydrolase, ${ }^{38}$ inosine 5'-monophosphate dehydrogenase, 39 and numerous nucleobase sulfurizing enzymes. ${ }^{21}$ In these cases C-S bond formation is followed by elimination of a halide, a phosphate, or a hydride from the ipso-position. 39 In contrast, EanB removes a proton resulting in oxidative sulfur transfer. We are unaware of any precedence for this reaction type in enzymology. ${ }^{1}$ On the other hand, the EanBcatalyzed reaction is somewhat reminiscent of peracid-mediated epoxidation of olefins (Scheme 2). $4^{40}$ This reaction mediates oxidative $\mathrm{C}-\mathrm{O}$ bond formation with reductive $\mathrm{O}-\mathrm{O}$ bond cleavage. However, because of the high pKa of peracids and because isolated olefins are electron rich, the reaction is believed to occur via nucleophilic attack by the $\mathrm{sp}^{2}$ carbon.
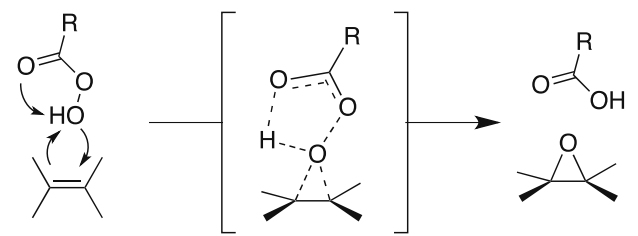

Scheme 2.

Active site conservation among YnjE rhodaneses. The structure of EanB provides indications that many rhodanese-like enzymes with currently unknown function might catalyze related reactions. EanB belongs to a large group of enzymes termed the YnjE rhodaneses group, because YnjE from $E$. coli was the first structurally characterized representative of this family. ${ }^{24}$ Members of this class occur in several bacterial phyla (proteobacteria, spirochaetes, fusobacteria and firmicutes) and several archaeal species. YnjE rhodaneses are characterized by a highly conserved six-residue active site motif (CG[T/S]GWR, Cys412 - Arg417 in EanB). ${ }^{24}$ Based on structural evidence we can now attribute specific roles to each of these residues (Figure 4). Cys412 is responsible to present the sulfane sulfur in the active site. The backbone of Gly413 hydrogen bonds to the thiolate side chain of Cys412 in the apo form. The C $\alpha$ atom of Gly413 packs so tightly to Tyr353 that any other residue in this position would cause displacement of the catalytic base. Hence, Gly is conserved at this position. The essential catalytic role of Thr414 has been discussed above. Gly415 adopts a backbone conformation $(\phi$ : $134^{\circ} ; \psi:-14^{\circ}$ ) that is difficult to attain for any other residue. The indole ring of Trp416 contributes a large hydrophobic patch to the substrate binding pocket, interacting with the imidazole ring, the $\beta$ carbon and one of the $\mathrm{N}-\alpha$-methyl groups.

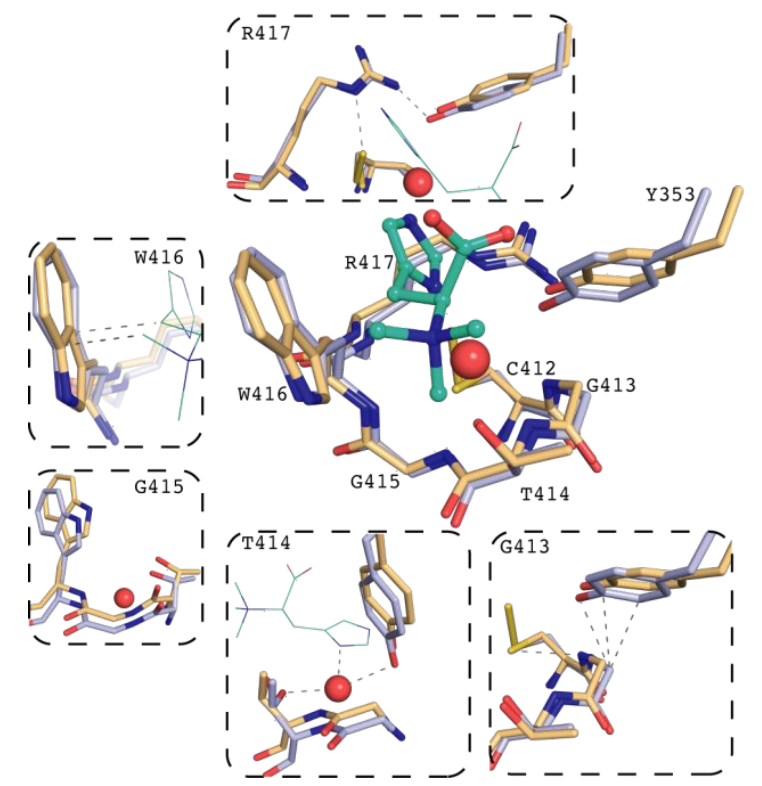

Figure 4: Overlay of the conserved active site motif (CG[T/S]GWR) in EanB (pale blue) and YnjE (pale orange). The residue numbering is according to EanB from C.limicola.

Finally, Arg417 hydrogen bonds to Tyr353 and the persulfide anion on Cys412. Strict conservation of a Thr or Ser at position 414 is particularly interesting because this residue makes no direct contact with the substrate or catalytic residues. Instead, the $\beta$ hydroxy side chain of Thr414 forms a strong hydrogen bond to $\mathrm{H}_{2} \mathrm{O} \_1$, which in turn also forms 
hydrogen bonds to Tyr353 and TMH. The observation that $\operatorname{EanB}_{\mathrm{T}_{414} \mathrm{~A}}$ is at least two orders of magnitude less active than wild type demonstrates that $\mathrm{H}_{2} \mathrm{O} \_1$ is crucial for catalysis. As described above, the most likely role of $\mathrm{H}_{2} \mathrm{O} \_1$ is to mediate proton transfer between Tyr353 and TMH ( $3 \mathbf{a} \rightarrow \mathbf{3 b}$, Scheme 1). In this way Thr414 and $\mathrm{H}_{2} \mathrm{O}_{-} 1$ contribute to substrate activation.

In addition to the conserved active site motif (CG[T/S]GWR), YnjE rhodaneses also share other active site features that are essential for EanB activity. Tyr353 and Tyr355 are both strictly conserved. Mutation of Tyr353 produced a completely inactive EanB variant, corroborating the key role of this residue in catalysis. Mutation of Tyr355 predominantly affected substrate binding, which is consistent with the observed contact of this residue with the carboxylate of TMH. Finally, the ammonium binding pocket formed by Tyr188, Trp216, Trp416, Tyr355 and Glu211 is also largely conserved.

On the other hand, EanB also contains features that are exclusive to homologs that are involved in ergothioneine biosynthesis." The most important distinction from other YnjE rhodaneses is an extra active site loop in EanB that folds over the TMH binding pocket (Figure $3 \mathrm{~B}$, residues $245-260$ ). Secondly, Tyr375 is strictly conserved among EanB homologs, but is absent in several subgroups of YnjE rhodaneses. Mutation of this residue in EanB reduced the substrate affinity dramatically, suggesting that Tyr375 is a determinant for substrate specificity.

This analysis shows that most active site residues with a role in substrate-binding and catalysis by EanB are conserved in all YnjE rhodaneses. Therefore, we predict that most YnjE rhodaneses catalyze oxidative sulfurization of heterocycles with a quaternary ammonium moiety. However, we should note that YnjE from E. coli has already been attributed a function in molypterin biosynthesis as a sulfur transferase..$^{11}$ This activity does not involve oxidative C-S bond formation and may therefore be a secondary function of YnjE.

Conclusions. In this report we describe the crystal structure of the rhodanese-like enzyme EanB. This enzyme catalyzes oxidative sulfurization of TMH as the second step in oxygen-independent ergothioneine biosynthesis in C. limicola. Structural and kinetic evidence suggest that this reaction occurs via nucleophilic attack of an active site cysteine persulfide anion on the imidazolium ring of TMH. This rate-limiting step is followed by basecatalyzed deprotonation and rearomatization of the mercaptoimidazole side chain of the product ergothioneine. Conservation of key active site residues across all members of the YnjE rhodanese enzyme family suggest that this novel reaction type may apply to several other biosynthetic pathways of heretofore unknown sulfur metabolites.

\section{ASSOCIATED CONTENT}

Supporting Information. Detailed descriptions of all experiments, supporting figures $\left(\mathrm{S}_{1}-\mathrm{S}_{15}\right)$ and tables $\left(\mathrm{S}_{1}-\mathrm{S}_{3}\right)$ are shown in the supporting information. The Supporting Information is available free of charge as a PDF file on the ACS Publications website.

\section{AUTHOR INFORMATION}

\section{Corresponding Author}

florian.seebeck@unibas.ch

\section{Author Contributions}

\$These authors contributed equally.

\section{Funding Sources}

European Research Council (ERC-2013- StG 336559) Swiss National Science Foundation, Commission for Technology an Innovation

\section{Notes}

The authors declare no competing financial interest.

\section{ACKNOWLEDGMENT}

The authors would like to thank Alice Maurer for HR ESI MS measurements. This project was supported by the Swiss National Science Foundation, the University of Basel, the Commission for Technology an Innovation, the "Professur für Molekulare Bionik" and a starting grant from the European Research Council (ERC-2013-StG 336559). We thank the DESY PETRAIII (Hamburg, Germany) pil and the SLS (Villigen, Switzerland) Xo6DA for access to facilities and beamline stuff for support.

\section{REFERENCES}

1. Dunbar, K. L.; Scharf, D. H.; Litomska, A.; Hertweck, C., Enzymatic Carbon-Sulfur Bond Formation in Natural Product Biosynthesis. Chem. Rev. 2017, 117 (8), 5521 - 5577.

2. Schöneich, C., Sulfur Radical-Induced Redox Modifications in Proteins: Analysis and Mechanistic Aspects. Antioxid Redox Signal 2017, 26 (8), 388 - 405.

3. Bonfio, C.; Valer, L.; Scintilla, S.; Shah, S.; Evans, D. J.; Jin, L.; Szostak, J. W.; Sasselov, D. D.; Sutherland, J. D.; Mansy, S. S., UV-light-driven prebiotic synthesis of iron-sulfur clusters. Nat. Chem. 2017, 9 (12), 1229 - 1234.

4. Filipovic, M. R.; Zivanovic, J.; Alvarez, B.; Banerjee, R., 
Chemical Biology of $\mathrm{H}_{2} \mathrm{~S}$ Signaling through Persulfidation. Chem. Rev. 2018, 118 (3), 1253 - 1337.

5. Fass, D.; Thorpe, C., Chemistry and Enzymology of Disulfide Cross-Linking in Proteins. Chem. Rev. 2018, 118 (3), $1169-1198$.

6. Baldwin, J. E.; Bradley, M., Chem. Rev. 1990, 9o, 1079 - 1088.

7. Jarrett, J. T., The biosynthesis of thiol- and thioethercontaining cofactors and secondary metabolites catalyzed by radical S-adenosylmethionine enzymes. J Biol Chem. 2015, 290 (7), 3972 - 3979.

8. Jurgenson, C. T.; Begley, T. P.; Ealick, S. E., The Structural and Biochemical Foundations of Thiamin Biosynthesis. In Annu. Rev. Biochem., 2009; Vol. 78, pp 569-603.

9. Chatterjee, A.; Abeydeera, N. D.; Bale, S.; Pai, P.-J.; Dorrestein, P. C.; Russell, D. H.; Ealick, S. E.; Begley, T. P., Saccharomyces cerevisiae THI $4 \mathrm{p}$ is a suicide thiamine thiazole synthase. Nature 2o11, 478 (7370), 542-U146.

10. Eser, B. E.; Zhang, X.; Chanani, P. K.; Begley, T. P., From Suicide Enzyme to Catalyst: The Iron-Dependent Sulfide Transfer in Methanococcus jannaschii Thiamin Thiazole Biosynthesis. J. Am. Chem. Soc. 2016, 138 (11), 3639 - 3642.

11. Burn, R.; Misson, L. E.; Meury, M.; Seebeck, F. P., Anaerobic Origin of Ergothioneine. Angew Chem Int Ed Engl. 2017, 56 (41), 12508 - 12511.

12. Goncharenko, K. V.; Vit, A.; Blankenfeldt, W.; Seebeck, F. P., Structure of the Sulfoxide Synthase EgtB from the Ergothioneine Biosynthetic Pathway. Angew. Chem. Int. Ed. Engl. 2015, 54 (9), 2821 - 2824.

13. Vit, A.; Mashabela, G. T.; Blankenfeldt, W.; Seebeck, F. P., Structure of the Ergothioneine-Biosynthesis Amidohydrolase EgtC. ChemBioChem 2015, 16 (10), 1490 1496.

14. Song, H.; Hu, W.; Naowarojna, N.; Her, A. S.; Wang, S. G.; Desai, R.; Qin, L.; Chen, X.; Liu, P., Mechanistic studies of a novel C-S lyase in ergothioneine biosynthesis: the involvement of a sulfenic acid intermediate. Sci. Rep. 2015, 5. $(11870)$.

15. Liao, C.; Seebeck, F. P., Convergent Evolution of Ergothioneine Biosynthesis in Cyanobacteria. Chembiochem 2017, 18 (21), 2115 - 2118.

16. Seebeck, F. P., In vitro reconstitution of Mycobacterial ergothioneine biosynthesis. J. Am. Chem. Soc. 2010, 132, 6632-6633.

17. Naowarojna, N.; Cheng, R.; Chen, L.; Quill, M.; Xu, M.; Zhao, C.; Liu, P., Mini-Review: Ergothioneine and Ovothiol Biosyntheses, an Unprecedented Trans-Sulfur Strategy in Natural Product Biosynthesis. Biochemistry 2018, 57 (24), $3309-3325$.

18. Peck, S. C.; van der Donk, W. A., Go It Alone: Four Electron Oxidations by Mononuclear Non-heme Iron Enzymes. J. Biol. Inorg. Chem. 2017, 22 (2 - 3), 381 - 394.

19. Faponle, A. S.; Seebeck, F. P.; de Visser, S. P., Sulfoxide Synthase versus Cysteine Dioxygenase Reactivity in a Nonheme Iron Enzyme. J. Am. Chem. Soc. 2017, 139 (27), 9259 - 9270.

20. Wei, W. J.; Siegbahn, P. E.; Liao, R. Z., Theoretical Study of the Mechanism of the Nonheme Iron Enzyme EgtB. Inorg. Chem. 2017, 56 (6), 3589- 3599.

21. Leimkühler, S.; Bühning, M.; Beilschmidt, L., Shared Sulfur Mobilization Routes for tRNA Thiolation and Molybdenum Cofactor Biosynthesis in Prokaryotes and Eukaryotes. Biomolecules 2017, 7 (1), E5.

22. Sasaki, E.; Zhang, X.; Sun, H. G.; Lu, M. Y.; Liu, M. Y.; Liu, T. L.; Ou, A.; Li, J. Y.; Chen, Y. H.; Ealick, S. E.; Liu, H. W., Co-opting sulphur-carrier proteins from primary metabolic pathways for 2-thiosugar biosynthesis. Nature 2014, 510 (7505), 427-431.
23. Wright, C. M.; Christman, G. D.; Snellinger, A. M.; Johnston, M. V.; Mueller, E. G., Direct evidence for enzyme persulfide and disulfide intermediates during 4-thiouridine biosynthesis. Chem. Commun. 2006, 29, 3104-3106.

24. Hänzelmann, P.; Dahl, J. U.; Kuper, J.; Urban, A.; MüllerTheissen, U.; Leimkühler, S.; Schindelin, H., Crystal structure of YnjE from Escherichia coli, a sulfurtransferase with three rhodanese domains. Protein. Sci. 2009, 18 (12), 2480 - 2491.

25. Goldschmidt, L.; Cooper, D. R.; Derewenda, Z. S.; D., E., Toward rational protein crystallization: A Web server for the design of crystallizable protein variants. Protein Sci. 2007, 16 (8), 1569 - 1576.

26. Long, F.; Vagin, A. A.; Young, P.; Murshudov, G. N., BALBES: a molecular-replacement pipeline. Acta Crystallogr D Biol Crystallogr. 20o8, 64, 125 - 132.

27. Dougherty, D. A., The Cation $\pi$ Interaction. Acc Chem Res 2013, 46 (4), 885 - 893.

28. Mahadevi, A. S.; Sastry, G. N., Cation $-\pi$ Interaction: Its Role and Relevance in Chemistry, Biology, and Material Science. Chem. Rev. 2013, 113 (3), 2100 - 2138.

29. Ziegler, C.; Bremer, E.; Krämer, R., The BCCT family of carriers: from physiology to crystal structure. Mol Microbiol. 2010, 78 (1), 13 - 34 .

30. Steudel, R.; Steudel, Y., Derivatives of cysteine related to the thiosulfate metabolism of sulfur bacteria by the multienzyme complex "Sox"-studied by B3LYP-PCM and G3X(MP2) calculations. Phys Chem Chem Phys. 2010, 12 (3), $630-644$.

31. Amyes, T. L.; Diver, S. T.; Richard, J. P.; Rivas, F. M.; Toth, K., Formation and Stability of N-Heterocyclic Carbenes in Water: The Carbon Acid pKa of Imidazolium Cations in Aqueous Solution. J. Am. Chem. Soc. 2004, 126 (13), 4366 4374 .

32. Cipollone, R.; Ascenzi, P.; Visca, P., Common Themes and Variations in the Rhodanese Superfamily. IUBMB Life 2007, 59 (2), $51-59$.

33. Park, C. M.; Weerasinghe, L.; Day, J. J.; Fukuto, J. M.; Xian, M., Persulfides: current knowledge and challenges in chemistry and chemical biology. Mol. Biosyst. 2015, 11 (7), $1775-1785$.

34. Breslow, R., On the Mechanism of Thiamine Action. Evidence from Studies on Model Systems. J. Am. Chem. Soc. 1958, 8 o (14), 3719 - 3726.

35. Meyer, D.; Neumann, P.; Ficner, R.; Tittmann, K., Observation of a stable carbene at the active site of a thiamin enzyme. Nat. Chem. Biol. 2013, 9, 488 - 490.

36. Retey, J., The urocanase story: a novel role of NAD+ as electrophile. Arch Biochem Biophys. 1994, 314 (1), 1 - 16.

37. Graminski, G. F.; Zhang, P. H.; Sesay, M. A.; Ammon, H. L.; Armstrong, R. N., Formation of the 1-(S-glutathionyl)-2,4,6trinitrocyclohexadienate anion at the active site of glutathione S-transferase: evidence for enzymic stabilization of sigma-complex intermediates in nucleophilic aromatic substitution reactions. Biochemistry 1989, 28 (15), $6252-6258$.

38. Johnson, C. M.; Monzingo, A. F.; Ke, Z.; Yoon, D. W.; Linsky, T. W.; Guo, H.; Robertus, J. D.; Fast, W., On the mechanism of dimethylarginine dimethylaminohydrolase inactivation by 4-halopyridines. J. Am. Chem. Soc. 2011, 133 (28), 10951 - 10959.

39. Hedstrom, L., IMP Dehydrogenase: Structure, Mechanism, and Inhibition. Chem. Rev. 2009, 109, 2903 - 2928.

40. Clayden, J.; Greeves, N.; Warren, S., Organic Chemistry. second edition ed.; Oxford University Press Inc.: Oxford, 2012. 
41. Dahl, J. U.; Urban, A.; Bolte, A.; Sriyabhaya, P.; Donahue, J. L.; Nimtz, M.; Larson, T. J.; Leimkühler, S., The identification of a novel protein involved in molybdenum cofactor biosynthesis in Escherichia coli. J Biol Chem. 2011, 286 (41), $35801-35812$. 


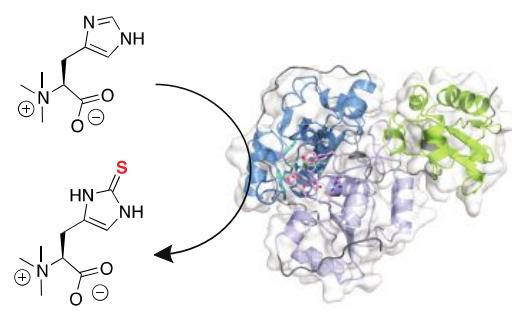

